\title{
Long distance communication in the human brain: timing constraints for inter-hemispheric synchrony and the origin of brain lateralization
}

\author{
FRANCISCO ABOITIZ, JAVIER LÓPEZ and JUAN MONTIEL
}

Departamento de Psiquiatría and Centro de Investigaciones Médicas, Facultad de Medicina, Pontificia Universidad Católica de Chile, and Millenium Nucleus for Integrative Neuroscience, Santiago, Chile

\begin{abstract}
Analysis of corpus callosum fiber composition reveals that inter-hemispheric transmission time may put constraints on the development of inter-hemispheric synchronic ensembles, especially in species with large brains like humans. In order to overcome this limitation, a subset of large-diameter callosal fibers are specialized for fast interhemispheric transmission, particularly in large-brained species. Nevertheless, the constraints on fast interhemispheric communication in large-brained species can somehow contribute to the development of ipsilateral, intrahemispheric networks, which might promote the development of brain lateralization.
\end{abstract}

Key terms: axon, brain lateralization, corpus callosum, inter-hemispheric transfer, synchrony, vision

\section{INTRODUCTION}

In this paper we will refer to some aspects of our own work which bear upon the issues raised by the late Francisco Varela, namely those related to brain synchrony and largescale communication in the nervous system (Varela, 1995; Rodríguez et al., 1999; Varela et al., 2001). In this perspective, distributed neuronal networks, defined as such by synchronized oscillations in activity, are fundamental to the generation of unified cognitive phenomena. For synchronized oscillations to occur among distant neurons, they must be interconnected by very fast reciprocal interactions, so that the phase of the oscillations is not significantly delayed by the effects of neuronal transmission (Singer, 1999).

From a neuroanatomical point of view, the increasingly long distances between cerebral regions as brains become larger may place constraints on the development of large-scale synchronic neuronal ensembles. In addition to the time involved in synaptic transmission, there is also the development of increasingly long axons to communicate with distant regions of the brain. For example, in a mouse, interhemispheric distance can be as short as 10 $\mathrm{mm}$, while in humans, axons must grow over $130 \mathrm{~mm}$ or more to connect the two hemispheres. If, for example, human callosal fibers have a conduction velocity of $5 \mathrm{~m} / \mathrm{sec}$, the delay in inter-hemispheric transmission time can be about $26 \mathrm{msec}$, which is about the duration of a whole 40 $\mathrm{Hz}$ cycle. This phase delay might be functionally deleterious, especially for reciprocally connected inter-hemispheric networks in which the delay begins to accumulate after successive trips along the corpus callosum. Therefore, size does matter when it comes to constructing largescale synchronic ensembles.

Recent studies have suggested that in some instances, inter-hemispheric communication can be constrained by increasingly long transmission delays as the distance between the hemispheres increases in species with large brains 
(Olivares et al., 2001). Furthermore, it has been postulated that limitations of this sort may favor local processing within each hemisphere, in this way promoting the development of brain lateralization (Ringo et al., 1994). At the end of the paper, we will discuss this possibility, especially in relation to large-scale intrahemispheric projections from the temporoparietal regions to the prefrontal lobe, which are involved in higher-order cognitive phenomena such as language and working memory.

INTER-HEMISPHERIC COMMUNICATION AND BRAIN SIZE

A few years ago, Ringo et al. (1994) proposed a highly controversial hypothesis for the origin of brain lateralization in the human brain. Their argument was based on the fact that, as the human brain becomes larger, so does the distance between the cerebral cortices of the two sides (especially considering that fibers usually follow a rather convoluted pathway instead of running straight to the other hemisphere). The resulting delay in inter-hemispheric transmission might pose an important constraint for inter-hemispheric communication, which would promote the development of hemispheric specialization for specific tasks. At the same time, it has been found that the number of callosal axons in relation to total cortical cells tends to diminish in large-brained species (Rilling and Insel, 1999; Olivares et al. 2000, 2001), and that callosal size (especially some callosal segments) tends to decrease in those human subjects that are anatomically or functionally more lateralized (Witelson, 1985, 1989; Aboitiz, 1992; Aboitiz et al., 1992a, b; 1995; Dorion et al., 2000). Therefore, it is possible that in species with large brains, there are some constraints limiting the degree to which synchronic ensembles may involve both hemispheres.

Nevertheless, inter-hemispheric synchronization has been demonstrated in the visual cortex of the cat (Engel et al., 1991), and more recently, in the human brain (Rodríguez et al. 1999), indicating that at least in some domains synchronous activity may spread across the hemispheres. The question we want to ask at this point is as follows: if interhemispheric synchronization is possible in the human brain, can this be mediated by any kind of callosal fiber, or must it be mediated by specialized "callosal channels" represented by a distinct subset of fiber groups? If the first alternative is correct, this might imply that inter-hemispheric communication might not be a problem in large brains. However, , we consider that evidence tends to favor the second possibility, which is that there may be specialized fiber systems involved in the generation of synchronic, inter-hemispheric ensembles.

FIBER COMPOSITION OF THE CORPUS CALLOSUM AND VISUAL CALLOSAL CONNECTIONS

Analyses of fiber composition in the primate corpus callosum reveal a wide variety of fiber calibers, which are distributed in an uneven manner along this structure (see Fig. 1; La Mantia and Rakic, 1990; Aboitiz et al., 1992b). Callosal regions connecting prefrontal and temporoparietal association areas are characterized by large proportions of low myelinated, small caliber, slowconducting fibers, while in regions connecting primary and secondary sensorimotor areas, there is a concentration of highly-myelinated, large-caliber (more than $3 \mathrm{Bm}$ in diameter), fast-conducting fibers. (Nevertheless, these large-diameter fibers make up a small proportion of the total fibers; less than $1 \%$; Aboitiz et al., 1992b.) In the human, the most common fiber diameter observed is about $0.8 \mu \mathrm{m}$ (and this may be an overestimate; see below) and the maximal fiber sizes can be up to 10 $\mu \mathrm{m}$ in diameter. Assuming that the relationship between fiber diameter and conduction velocity is similar in peripheral nerves and in the central nervous system, we can make some educated guesses about the fiber velocities based on peripheral data on nerves (Aboitiz et al., 1992b). Thus, the most common myelinated callosal fibers of $0.8 \mu \mathrm{m}$ in diameter may have an estimated 
conduction velocity of $10 \mathrm{~m} / \mathrm{sec}$, with an inter-hemispheric delay of around $13 \mathrm{msec}$, about half of a $40 \mathrm{~Hz}$ cycle (which lasts 25 msec). This undoubtedly poses difficulties for inter-hemispheric synchronic oscillations at $40 \mathrm{~Hz}$. In this case, the only possibility for synchronic firing at this frequency is synchronous thalamic activation, perhaps driven by sensory input. On the other hand, the far less abundant 3 $\mu \mathrm{m}$-caliber fibers may conduct at velocities of $40 \mathrm{~m} / \mathrm{sec}$, with inter-hemispheric delays of $3.25 \mathrm{msec}$, which makes $40 \mathrm{~Hz}$ synchronic activity more plausible. In the monkey, antidromic inter-hemispheric transmission times between areas V1/V2 of the two cerebral hemispheres range between 2.6 and $18 \mathrm{msec}$ (Innocenti et al. 1995), with conduction velocities of 18 and
$2.6 \mathrm{~m} / \mathrm{sec}$ (with $45-47 \mathrm{~mm}$ of interhemispheric distance), respectively, which more or less fits the human estimates. In the human, the visually evoked potential inter-hemispheric latency has been calculated at around 13 or $26 \mathrm{msec}$. However, human measures are taken transcranially, and the delays obtained may include many other factors beside axonal conduction times.

The uneven distribution of fiber types in different callosal regions suggests important functional differences in interhemispheric communication between different types of cortical areas. First, it is important to recall that perhaps many callosal projections are homotopic, that is, they connect equivalent regions of each hemisphere. This is not to say that there
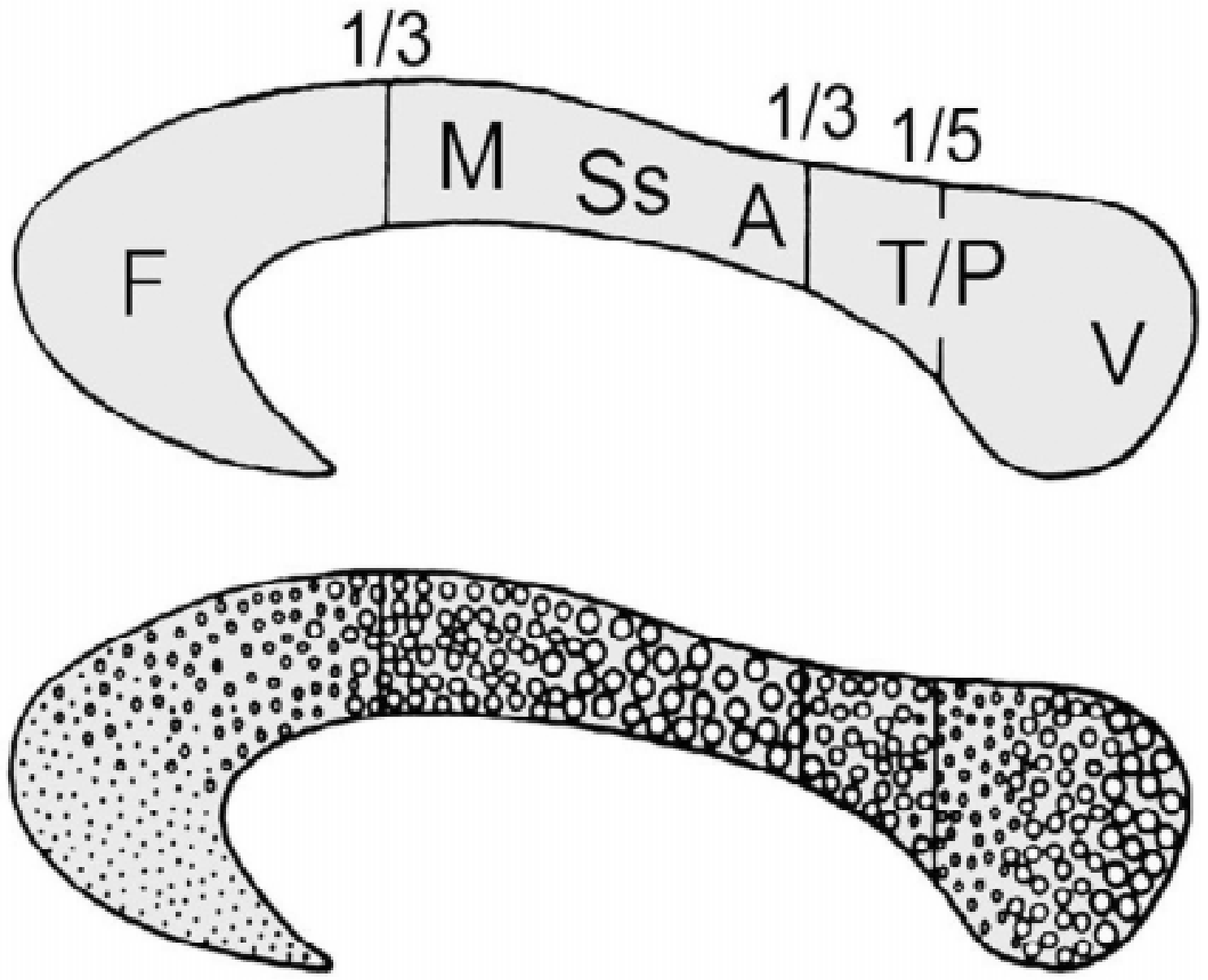

Figure 1. Diagrams indicating a cross-section of the human corpus callosum, with a rough map of the representation of different cortical areas (above) and a scheme illustrating the spatial distribution of fibers according to their diameter. F, frontal; M, motor; Ss, somatosensory; A, auditory; T/P, temporal/parietal; $\mathrm{V}$, visual. 
are no heterotopic callosal projections (i.e. connecting different areas across the hemispheres). Importantly, callosal cells and fibers connecting lower-order visual areas (V1, V2, V3) with a visuotopic organization (with a topographic map of the spatial relations in the retinal image) tend to be concentrated in the borders between these areas. More specifically, most callosal-projecting cells and their terminals are located in a stripe corresponding to the location of the visual field's midline; these cells can be found both in superficial and in deep cortical layers (Innocenti, et al.,1995). Since each hemisphere contains a representation of the contralateral visual hemifield, callosal fibers serve to connect the two hemirepresentations of the whole visual field at the level of the midline. In these same visual areas, a few cells in the deep layer $\mathrm{V}$ have been found to project through the callosum from regions well within the respective areas, representing peripheral regions of the visual field. The explanation for the presence of these cells is not yet clear, but may relate to anticipation of trajectories of fast-moving objects from one visual field to the other (see below).

A similar situation occurs in the somatosensory cortex, S1, and the motor cortex, M1 (see Innocenti, 1986). There, most callosal-projecting cells are restricted to a narrow band representing the body midline. In auditory primary and secondary areas of the rat, which have a detailed tonotopic organization (i.e. topographic to the cochlea), callosal cells have been described to be concentrated in binaural bands, oriented in the same direction as the tonotopic representation so that each band has a full representation of the spectrum of auditory frequencies (Pallas et al. 1999; Pallas 2001). However, a previous report on the cat found that patches of callosal connections correlated with domains defined by ear dominance (Imig and Bruge, 1978), and other reports on the gerbil indicate that callosal axons and terminals aggregate in distinct fiber bundles parallel to isofrequency contours (Thomas et al., 1993; Scheich et al., 1993). Our previous findings indicate that large-caliber callosal fibers tend to be concentrated in regions connecting these primary and secondary sensorimotor areas, for which at least some transfer functions may need to be executed with a minimal transmission delay.

Perhaps the sensory system for which there is more detailed knowledge of the organization of callosal connections is the visual system. On the border between the primary and secondary visual areas of the cat and other mammals, there is a band containing two mirror images (one in V1 and the other in V2) of the central, binocular visual field; each image has an ipsilateral and a contralateral representation of the central visual field (Fig. 2; Olavarría, 1996). Callosal cells tend to connect points in the ipsilateral representation of one hemisphere with visually equivalent points in the contralateral representation of the other hemisphere (Olavarría, 1996). Furthermore, callosal cells correspond to cytochrome

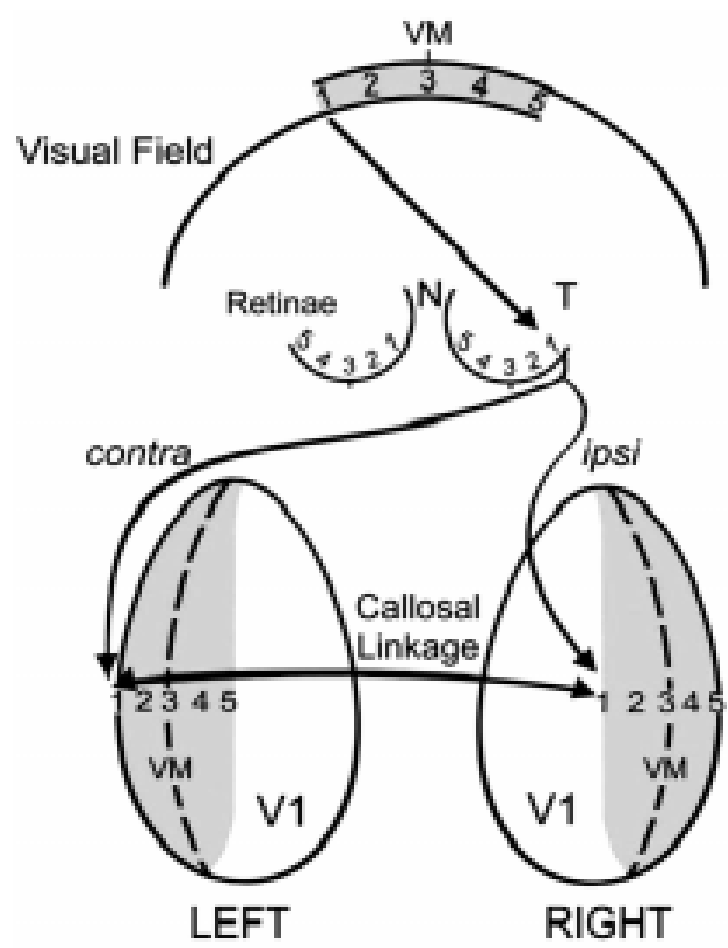

Figure 2. Diagram indicating the topographic representation of the midline visual field in area V1 in both hemispheres, and the arrangement of callosal connections in this region. $\mathrm{N}$, nasal retina; $\mathrm{T}$, temporal retina; VM, vertical meridian; V1, primary visual area. Shaded area in V1 indicates representation of the visual midline. Based on Olavarría (2001). 
oxidase-rich blobs in V1 (Boyd and Matsubara, 1991) with thick and thin cytochrome oxidase-rich stripes in V2 (Olavarría and Abel, 1996), and perhaps more interesting, with contralateral oculardominance columns in the ipsilateral representation of $\mathrm{V} 1$, and with ipsilateral ocular dominance columns in the contralateral representation of V1 (see Fig. 2; Olavarría, 2001). Developmentally, since ocular dominance columns are established before inter-hemispheric connections, this pattern is most likely explained in terms of the synchronous firing of ipsi and contralateral retinal collaterals, which produce the simultaneous activation of contralateral ocular dominance columns in one hemisphere and ipsilateral columns in the other. This may facilitate the establishment of callosal connections between contralateral and ipsilateral columns (Olavarría, 2001).

Callosal projections in higher-order cortical regions have been studied primarily in the prefrontal and the temporoparietal visual areas. These regions tend to be connected inter-hemispherically by lowly myelinated, thin diameter, slow-conducting fibers (La Mantia and Rakic, 1990; Aboitiz et al., 1992a). They tend to be distributed throughout the respective cortical regions, interdigitating with ipsilateral corticocortical projections and with thalamic projections in a manner resembling the ocular-dominance array of the primary visual cortex. These higher-order cortical areas tend to have a poor visuotopic organization; their cells have large receptive fields and respond better to stimulus properties and behavioral relevance than strictly to position in the visual field (Innocenti, 1986).

FUNCTIONS OF CALLOSAL FIBERS CONNECTING PRIMARY AND SECONDARY SENSORY AREAS

Depth perception. The concentration of fast-conducting, large-diameter fibers in primary/secondary sensorimotor areas might reflect the importance of performing the fusion of the two sensory and motor spatial hemirepresentations across the hemispheres. This is especially important in two sensory systems: the auditory system, which may use binaural cells to create a cortical sound localization system (sound localization is performed also at subcortical levels), and the visual system, in which central vision involves high-resolution processing and is related to aspects of depth perception.

The role of the corpus callosum in central vision, and more importantly, in depth perception has been debated for some time (Hubel, 1988). The interplay of ipsilateral and contralateral visual projections in the visual cortex has been considered to be fundamental for stereoscopic vision. Section of the optic chiasm, which cuts ipsilateral visual projections, consequently eliminating binocular vision in most of the visual field, also produces blindness in the lateralmost regions of the visual field, as well as in central points which are farther away from fixation. It also produces stereoblindness (inability to judge depth) in most of the visual field. However, points that are in front of the fixation point (closer to the observer) are visible and importantly, it has been described that depth judgement exists for these points provided the corpus callosum is intact. Damage of the posterior corpus callosum (through which visual fibers cross to the other hemisphere) results in inability to judge depth at these points in patients with damage to the optic chiasm (see Hubel, 1988). Following surgical callosal transection in newborn kittens, alterations in eye alignment have been reported (Payne et al., 1981). Furthermore, if performed sufficiently early in life (19 days postnatal), posterior callosal transection produces a reduction in the proportion of binocular neurons in the V1/ V2 border, something that is not observed if surgery is performed later in life (Elberger and Smith, 1985). Other studies indicate that in cats, the corpus callosum contributes to generating disparity sensitivity in superior cortical layers (Gardner and Cynader, 1987). In addition, experimentally induced early strabismus leads to an expanded callosal receiving zone in areas 17 and 18; in these conditions callosal cells also show decreased binocularity, decreased 
ability to respond to fast-moving stimuli, a small receptive field size and poor orientation selectivity (Milleret and Houzel, 2001). In acallosal humans, midline visual deficits (Jeeves, 1991), and impairment to inter-hemispheric depth comparisons when using head movements to determine relative depth (Rivest et al., 1994) have been reported.

In contrast to this evidence, other reports indicate that there are no behavioral deficits in depth perception in surgically acallosal kittens who underwent surgery at 11 days of age (Tinney et al., 1985), and that stereoscopic vision is mostly determined by the interactions between the thalamocortical crossed and uncrossed pathways in the visual cortex of each hemisphere, rather than by callosal interactions (Lepore et al., 1986; Ptito et al., 1991; Cowey and Wilkinson, 1991). It is possible that the corpus callosum is involved in more subtle mechanisms of depth perception than stereopsis (which is defined specifically as perception of binocular disparity to generate depth judgements), such as relative motion or parallax (that is, using the differences in relative motion of near and far objects to judge depth; Rivest et al., 1994). In this context, the finding that in primates, cortical area MT (an area sensitive to motion and intensely myelinated) may participate in stereoscopic vision (DeAngelis et al., 1998) is of the highest interest.

Recently, we studied the fiber composition of the posterior corpus callosum (which houses a large proportion of visual fibers) in different species which differ in brain size, namely the mouse, the rabbit, the cat, the dog, the cow and the horse (Olivares et al., 2001). Our main findings confirm and extend previous findings in the macaque and the mouse (Schüz and Preissl, 1996), and can be summarized in three points: First, there is quite a conservative cross-species fiber composition in the corpus callosum (the most common fiber diameter is between 0.1 and $0.2 \mathrm{Bm}$. This is lower than the findings in the human, but can be explained by suboptimal fixation conditions in the human tissue, in which low-diameter fibers are destroyed; furthermore, this runs in favor of our argument, as the median fiber diameter in the human may be even smaller than considered above. Secondly, there is a small group of large-diameter fibers whose caliber tends to increase in species with larger brains. The third point of interest in our study is that the largest fiber diameters were larger than those expected from their brain size in species with frontal vision (i.e. the cat and the dog), as compared to species with more lateral vision (i.e. the mouse, the rabbit, the horse and the cow; see Fig. 3). This finding is consistent with the hypothesis that the large callosal fibers are related to some aspects of central vision or depth perception. Furthermore, area MT of primates - possibly involved in stereopsis (see above) - is a highly myelinized one, and callosal fibers emerging from this region may be highly myelinated as well.

Participation of callosal fibers in interhemispheric synchronic processes. Another, though not alternative explanation for the existence of such fast-conducting fibers is that they participate in generating interhemispheric ensembles of synchronized neural activity (in fact, depth perception may well be related to the establishment of synchronized neuronal ensembles). In this context, it has been suggested that visual callosal fibers participate in predicting trajectories of moving objects across the midline, and in generating binding mechanisms in the central visual field (Milleret and Houzel, 2001), which may be of direct relevance to the question of interhemispheric synchronic ensembles. One important requisite for the generation of synchronic ensembles is that the dispersion of spike training must be below $10 \mathrm{~ms}$ (Singer 1999; Engel et al. 2001). As we discussed earlier, the inter-hemispheric transmission time for fibers of a modal fiber diameter is just in this range. Therefore, in order to transmit safely synchronic oscillations from one hemisphere to the other, only large-diameter fibers may be sufficiently safe.

Perhaps these large-diameter fibers correspond to cells that collect synchronous activity in one hemisphere and send it to the other hemisphere to induce the generation of inter-hemispheric synchronic 


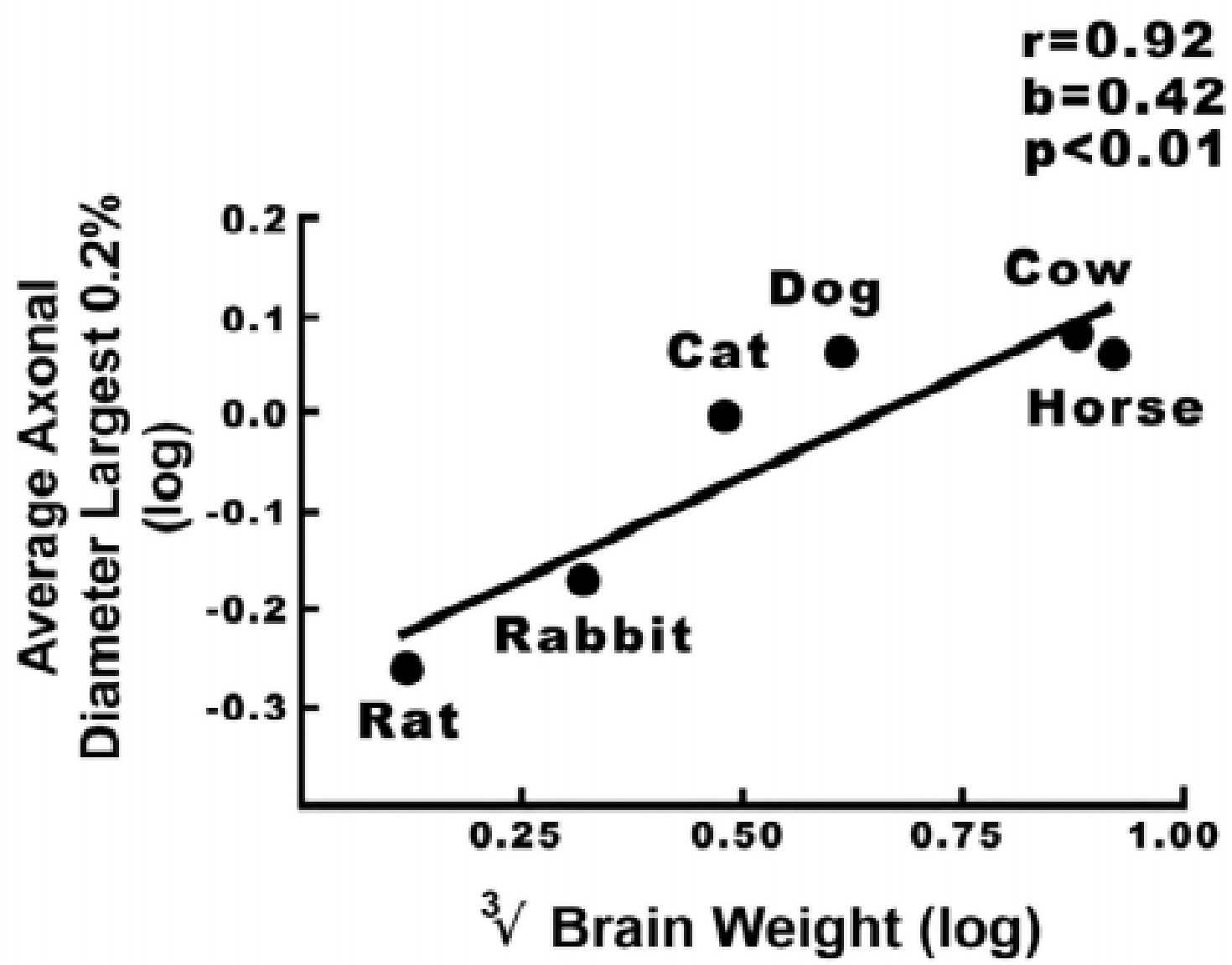

Figure 3. Log-log plot of maximum fiber sizes as a function of inter-hemispheric distance across species. Based on Olivares et al. (2001).

ensembles. Recently, a subset of pyramidal neurons has been found to be located in the superficial cortical layers (Gray and McCormick, 1996). These cells - termed "chattering cells" - intrinsically generate 20 - to- $70 \mathrm{~Hz}$ repetitive bursts firing in response to stimulation, and may serve as pacemakers for large, synchronized neuronal ensembles. One possibility would be that some of the large-diameter callosal fibers correspond to these chattering cells, which participate in coordinating neuronal ensembles in the two hemispheres.

Two points may be important in this context: one is that since large diameter fibers tend to be especially abundant in callosal regions connecting primary and secondary sensorimotor areas, this may relate to the establishment of interhemispheric synchrony at low levels in the hierarchy. On the other hand, regions connecting higher-order areas (especially prefrontal) have relatively few large-caliber fibers, and their modal diameter is well below $0.8 \mathrm{Bm}$. This may place some constraints on the possibility of establishing large-scale, high-level inter-hemispheric synchronic ensembles. It is possible that synchronic activity mediated by the callosum can be achieved only by specialized "channels" provided by just very few fast-conducting fibers. It is of interest that in all callosal regions, the proportions of large-diameter fibers in the corpus callosum tend to increase with age (Aboitiz et al., 1996; see also Godlewski, 1991). This may imply that these fibers participate in the generation of interhemispheric ensembles that become stabilized during late ontogeny.

Another point is that, although some large-diameter fibers tend to increase their 
caliber (and conduction velocity) in species with large brains (as mentioned, across species with different brain sizes, most callosal fibers tend to have a conservative diameter and degree of myelination), calculations suggest that the increase in fiber diameter may not be sufficient to fully compensate for the increasingly long inter-hemispheric distances. Thus, although the maximum fiber velocity increases in species with large brains, there is nonetheless an increase in the minimal interhemispheric transmission time (Olivares et al., 2001). Again, this may imply that, especially in large-brained species such as our own, there are particularly few specialized cells with fast-conducting axons that act as channels to establish synchronic activity across the hemispheres.

3. Evolution of the corpus callosum and corticocortical synchrony. The corpus callosum is the largest fiber tract in the mammalian brain, and exists exclusively in all placental mammals (in marsupials and monotremes, most inter-hemispheric connections run a long distance via the anterior commissure, while in nonmammals there are very few interhemispheric connections; Innocenti, 1986). Thus, the question arises about the adaptive value that the corpus callosum confers on placental mammals. We (Aboitiz and Ide, 1998) have proposed previously that interhemispheric fibers originated in mammals together with the appearance of the neocortex, and both were concomitant with the development of topographically organized sensory maps in the telencephalon (in reptiles, topographic sensory maps are restricted to mesencephalic levels, for which there is a well-developed tectal commissure). One type of inter-hemispheric design is that which is observed in marsupials, in which axons run to the other hemisphere through the anterior commissure. Another solution is that which evolved in placental mammals, with the corpus callosum originating from the hippocampal commissure. This may be a more efficient solution, since axons must travel a shorter distance between the hemispheres than if they crossed through the anterior commisssure.
Originally, the functions of callosal fibers may have been related to aspects of midline fusion, but in later stages they may have participated in spreading cortico-cortical synchronic activation across the midline, thus contributing to the generation of largescale, bihemispheric neuronal ensembles of corticocortical interactions. This is not to say that thalamocortical projections have no role in the establishment of cortical synchrony (Jones, 2001), but that the cortical spread of such assemblies may have been facilitated through a mechanism of inter-hemispheric reciprocal interaction. In this process, heterotopic callosal projections may be very important in propagating activity to other cortical areas. In a way, this may somehow be similar to the callosal propagation of an epileptic focus; inter-hemispheric reciprocal excitation works as a facilitating mechanism for the seizure, which spreads into ipsilateral territory in both hemispheres. In fact, in some patients callosotomy has proved to be an effective treatment to prevent the expansion of the epileptic activity. It is therefore possible that inter-hemispheric connections may be advantageous for the spread of normal synchronic activity in the cerebral cortex.

RELATIONSHIP TO LARGE-SCALE CORTICAL NETWORKS AND BRAIN LATERALIZATION

As mentioned, increased brain size across species and brain lateralization in humans are at least morphologically associated with a decrease in inter-hemispheric connectivity, both in terms of fiber numbers and of inter-hemispheric transmission delay. In this sense, although the corpus callosum may have contributed to the interhemispheric spread of synchronic ensembles in small mammals, it is perhaps possible that a point was reached in which beyond a given brain size, problems appeared for the transmission of some types of information between the hemispheres. Even considering that inter-hemispheric synchronic activity is possible in humans and other animals, the question still remains as to whether in our species there are certain 
subtle limiting factors in the generation of inter-hemispheric synchrony. These may have somewhat facilitated the development of strong, intrahemispheric neuronal loops, thus promoting the establishment of brain lateralization.

Fundamental elements in these ipsilateral neuronal loops are the large-scale temporoparietal-prefrontal reciprocal networks that participate in two important cognitive functions: language and working memory. Language depends on reciprocal networks connecting the superior temporal/ inferior parietal cortices (Wernicke's area) with the premotor cortices of the inferior frontal gyrus (Broca's area) in the left hemisphere. Working memory is a sort of conscious short-term memory that is used to maintain perceptual and long-term mnemonic information "on line" while performing a specific cognitive task. There are different types of working memory, which are based on specific anatomical networks. Working memory for objects depends on reciprocal connections between the inferior temporal gyrus and the ventrolateral prefrontal cortex, while working memory for spatial locations depends on parieto-dorsolateral prefrontal networks. Auditory working memory is related to networks similar to those involved in language. Based on this and other information, we (Aboitiz et al., 1995; Aboitiz and García, 1997a, b)* have proposed that language evolved as a specialization of a vocal-auditory working memory loop which initially participated in the generation and learning of increasingly complex vocal utterances. These utterances may have been fundamental for social behavior in early hominids. We further hypothesized that hemispheric specialization might have arisen from the consolidation of different types of temporoparietal-prefrontal networks in the two hemispheres, the left hemisphere emphasizing temporalprefrontal networks (such as those involved in language and object perception), while the right hemisphere emphasized parietoprefrontal networks (involved in visuospatial processing). The possibility that such ipsilateral neural networks evolved in relation to a decrease in interhemispheric communication needs to be further investigated.

There is not yet evidence for the existence of synchronic ensembles during performance in working memory tasks, but $40 \mathrm{~Hz}$ synchronic oscillations have been reported during linguistic performance (Engel et al., 2001). The role of synchronic activity in working memory processes, be they linguistic, auditory or visual, urgently needs to be investigated. The latest and fundamental contribution made by Rodríguez et al. (1999), in which synchronic activity was for the first time correlated with human conscious processing, precisely sets out the methodology for initiating these studies in humans. The task will be to determine asymmetries in the generation of synchronic ensembles in humans during different cognitive tasks, especially language and working memory. We consider that research on this subject, which was pioneered by Francisco Varela, will provide us with fundamental insights on the processes of brain lateralization, working memory, language and consciousness, and especially on the origins of the human mind.

\section{FINAL COMMENT}

In this paper, we have reviewed evidence suggesting that (i) there may be constraints to the generation of inter-hemispheric synchrony in species with large brains, and (ii) this may have somewhat facilitated the emergence of brain lateralization in the human brain (although other factors may have also been very important). We have also proposed that in its evolutionary origins, the corpus callosum may have participated in midline fusion tasks, and later as a bridge to spread synchronic activity, by establishing excitatory loops between the two hemispheres. However, as brains became larger, some kinds of synchronic processes may have become difficult to establish across the hemispheres (especially some relating to global networks involving higher-order areas), which led to the facilitation of intrahemispheric 
networks, promoting the development of brain lateralization. The work of Francisco Varela has provided us with a fundamental contribution by designing a new methodological paradigm by which hypotheses like this can be scientifically tested.

\section{ACKNOWLEDGMENTS}

This article is dedicated to the memory of Francisco Varela.

\section{REFERENCES}

ABOITIZ F (1992) Inter-hemispheric fiber systems and anatomical asymmetries in the human brain. Biol Res 25: 51-61

ABOITIZ F, GARCÍA RV (1997a) The evolutionary origin of the language areas in the human brain. A neuroanatomical perspective. Brain Res Rev 25: 381396

ABOITIZ, F., GARCÍA, R. (1997b) The anatomy of language revisited. Biological Research, 30: 171-183

ABOITIZ F, IDE A (1998) Anatomical asymmetries in language-related cortex and their relation to callosal function. In: STEMMER E, WHITAKER H (eds) Handbook of Neurolinguistics. New York: Academic Press. pp: 393-404

ABOITIZ F, SCHEIBEL A, ZAIDEL E (1992a) Morphometry of the sylvian fissure and the corpus callosum, with emphasis on sex differences. Brain 115: 121-1541

ABOITIZ F, SCHEIBEL AB, FISHER RS, ZAIDEL E (1992b) Fiber composition of the human corpus callosum. Brain Res 598: 143-153

ABOITIZ F, IDE A, NAVARRETE A, PEÑA M, RODRÍGUEZ E, WOLFF V, ZAIDEL E (1995) The anatomical substrate for language and hemispheric specialization. Biol Res 28:45-50

ABOITIZ F, RODRÍGUEZ E, OLIVARES R, ZAIDEL E (1996) Age-related changes in fiber composition of the human corpus callosum. Neuroreport 7: 17611764

BOYD JD, MATSUBARA JA (1991) The callosal pathway in cat visual cortex is patchy and orientation-specific. Soc Neurosci Abstr 17: 116

COWEY A, WILKINSON F (1991) The role of the corpus callosum and extra striate visual areas in stereoacuity in macaque monkeys. Neuropsychologia 29: 465-479

DE ANGELIS GC, CUMMING BG, NEWSOME WT (1998) Cortical area MT and the perception of stereoscopic depth. Nature 394: 677-680

DORION AA, CHANTOME M, HASBOUN D, ZOUAOUI A, MARSAULT C, CAPRON C, DUYME M (2000) Hemispheric asymmetry and corpus callosum morphometry: a magnetic resonance imaging study. Neurosci Res 36: 9-13

ELBERGER AJ, SMITH EL (1985) The critical period for corpus callosum section to affect cortical binocularity. Exp Brain Res 57: 213-223
ENGEL AK, KÖNIG P, KREITER AK, SINGER W (1991) Inter-hemispheric synchronization of oscillatory neural responses in cat visual cortex. Science 252: $1177-1179$

ENGEL AK, FRIES P, SINGER W (2001) Dynamic predictions: oscillations and synchrony in top-down processing. Nature Rev Neurosci 2: 704-716

GARDNER JC, CYNADER MS (1987) Mechanisms for binocular depth sensitivity along the vertical meridian of the visual field. Brain Res 413: 60-74

GODLEWSKI A (1991) Morphometry of myelin fibers in corpus callosum and optic nerve of aging rats. J Hirnforsch 83: 568-581

GRAY CM, MCCORMICK DA (1996) Chattering cells: superficial pyramidal neurons contributing to the generation of synchronous oscillations in the visual cortex. Science 274: 109-113

HUBEL DH (1988) Eye, Brain and Vision. New York: Scientific American Library. pp: 156-157

IMIG TJ, BRUGE JF (1978) Sources and terminations of callosal axons related to binaural and frequency maps in primary auditory cortex of the cat. J Comp Neurol 182: 637-660

INNOCENTI GM (1986) general organization of callosal connections in the cerebral cortex. In: JONES EG, PETERS A (eds) Cerebral Cortex, Vol. 5. New York: Plenum Press. pp: 291-354

INNOCENTI GM, AGGOUN-ZOUAOUI D, LEHMANN $P$ (1995) Cellular aspects of callosal connections and their development. Neuropsychologia 33: 961-988

JEEVES MA (1991) Stereo perception in callosal agenesis and partial callosotomy. Neuropsychologia 29: 19-34

JONES EG (2001) The thalamic matrix and thalamocortical synchrony. Trends Neurosci 24: 595-601

LA MANTIA AS, RAKIC P (1990) Cytological and quantitative characteristics of four cerebral commissures in the rhesus monkey. J Comp Neurol 291: 520-537

LEPORE F, PTITO M, LASSONDE M (1986) Stereoperception in cats following section of the corpus callosum and/or the optic chiasma. Exp Brain Res 61: 258-264

MILLERET C, HOUZEL JC (2001) Visual interhemispheric transfer to areas 17 and 18 in cats with convergent strabismus. Eur J Neurosci 13: 137-152

OLAVARRÍA JF (1996) Non-mirror symmetric patterns of callosal linkages in areas 17 and 18 in cat visual cortex. J Comp Neurol 366: 643-655

OLAVARRÍA JF (2001) Callosal connections correlate preferentially with ipsilateral cortical domains in cat areas 17 and 18 , and with contralateral domains in the 17/18 transition zone. J Comp Neurol 433: 441-457

OLAVARRÍA JF, ABEL PL (1996) The distribution of callosal connections correlates with the pattern of cytochrome oxidase stripes in visual area V2 of macaque monkeys. Cereb Cortex 6: 631-639

OLIVARES R, MICHALLAND S, ABOITIZ F (2000) Cross-species and intraspecies morphometric analysis of the corpus callosum. Brain, Behav Evol 55: 37-43

OLIVARES R, MONTIEL J, ABOITIZ F (2001) Species differences and similarities in the fine structure of the mammalian corpus callosum. Brain Behav Evol 57: 98-105

PALLAS SL, LITTMAN T, MOORE DR (1999) Crossmodal reorganization of callosal connectivity in auditory cortex without altering thalamocortical projections. Proc Natl Acad Sci (USA) 96: 8751-8756 
PALLAS SL (2001) Intrinsic and extrinsic factors that shape neocortical specification. Trends Neurosci 24: 417-425

PAYNE BR, BERMAN N, MURPHY EH (1981) A quantitative assessment of eye alignment in cats after corpus callosum transection. Exp Brain Res 43: 371376

PTITO M, LEPORE F, GUILLEMOT JP (1991) Stereopsis in the cat: behavioral demonstration and underlying mechanisms. Neuropsychologia 29: 443-464

RILLING JK, INSEL TR (1999) Differential expansion of neural projection systems in primate brain evolution. Neuroreport 10: 1453-1459

RINGO JL, DOTY RW, DEMETER S, SIMARD PY (1994) Time is of the essence: a conjecture that hemispheric specialization arises from inter-hemispheric conduction delay. Cereb Cortex 4: 331-343

RIVEST J, CAVANAGH P, LASSONDE M (1994) Interhemispheric depth judgement. Neuropsychologia 32: 69-76

RODRÍGUEZ E, GEORGE N, LACHAUX J-P, MARTINERIE J, RENAULT B, VARELA FJ (1999) Perception's shadow: long-distance gamma band synchronization of human brain activity. Nature 397: 430-433

SCHEICH H, HEIL P, LANGNER G (1993) Functional organization of auditory cortex in the Mongolian gerbil (Meriones unguiculatus). II. Tonotopic2Deoxyglucose. Eur J Neurosci 5: 898-914
SCHÜZ A, PREISSL H (1996) Basic connectivity of the cerebral cortex and some considerations on the corpus callosum. Neurosci Biobehav Rev 20: 567-570

SINGER W (1999) Neuronal synchrony: a versatile code for the definition of relations? Neuron 24: 49-65

THOMAS H, TILLEIN J, HEIL P, SCHEICH H (1993) Functional organization of auditory cortex in the Mongolian gerbil (Meriones unguiculatus). I. Electrophysiological mapping of frequency representation and distinction of fields. Eur J Neurosci 5: 882-897

TINNEY B, ELBERGER AJ, VANDEWATER ML (1985) Binocular depth perception in the cat following early corpus callosum section. Exp Brain Res 60: 19-26

VARELA FJ (1995) Resonant cell assemblies: a new approach to cognitive functions and brain synchrony. Biol Res 28: 81-95

VARELA FJ, LACHAUX J-P, RODRÍGUEZ E, MARTINERIE J (2001) The brainweb: phase synchronization and large-scale integration. Nature Rev Neurosci 2: 229-239

WITELSON SF (1985) The brain connection. The corpus callosum is larger in left-handers. Science 229: 665668

WITELSON SF (1989) Hand and sex differences in the isthmus and genu of human corpus callosum: a postmortem morphological study. Brain 112: 799835 